Tondorf, Karin, 2007, Fair P(I)ay - Entgeltgleichheit für Frauen und Männer. Hrsg. vom Bundesministerium für Familie, Senioren, Frauen und Jugend. Berlin

Tondorf, Karin/Jochmann-Döll, Andrea, 2008, Leistungsabhängige Entgeltdifferenzierung auf dem gleichstellungspolitischen Prüfstand. In: Krell, Gertraude (Hg.): Chancengleichheit durch Personalpolitik. Gleichstellung von Frauen und Männern in Unternehmen und Verwaltungen. 5. überarbeitete Auflage. Wiesbaden, S. 283-298
Winter, Regine, 2006, Die Regelung der Arbeits- und Wirtschaftsbedingungen durch Tarifnormen - Typischer Inhalt von Tarifvertragsnormen. In: Däubler, Wolfgang (Hg.): Tarifvertragsgesetz. 2. Auflage. Baden-Baden, S. 448-535

Winter, Regine,1998, Gleiches Entgelt für gleichwertige Arbeit. Ein Prinzip ohne Praxis. Baden-Baden

\title{
Deutsche Frauenpolitik auf ihr internationales Fundament stellen
}

Nicht nur 90 Jahre Frauenwahlrecht und 60 Jahre Art. 3 Abs. 2 GG, sondern auch auf internationaler Ebene gibt es dieses und nächstes Jahr einige Jubiläen zu feiern: 25 Jahre Pekinger Aktionsplattform, 10 Jahre Millenniumsgipfel ${ }^{1}$. Und schließlich wurde vor 30 Jahren das Übereinkommen der Vereinten Nationen zur Beseitigung jeder Form von Diskriminierung der Frau (CEDAW) ${ }^{2}$ abgeschlossen und im nächsten Jahr kann sein 25-jähriges Inkrafttreten in der Bundesrepublik ${ }^{3}$ gefeiert werden; eine gute Gelegenheit, die Forderungen an die deutsche Frauenpolitik für die nächste Legislaturperiode auf „völkerrechtliche Füße“ zu stellen. Dies umso mehr, weil über das CEDAW-Abkommen in der Öffentlichkeit und in der Politik kaum geredet wird, obwohl in diesem Jahr der 6. periodische Bericht der Bundesrepublik $^{4}$ vor dem CEDAW-Vertragsausschuss $^{5}$ verhandelt wurde. Dieses Abkommen, seine Bedeutung für das nationale Antidiskriminierungsund Gleichstellungsrecht und seine Möglichkeiten, es auch auf nationaler Ebene aktiv zu nutzen, sind in Deutschland nach wie vor zu wenig bekannt.

\section{CEDAW als wichtiges Instrument für die nationale Frauenpolitik}

CEDAW ist ein internationales Abkommen, das alle gleichstellungsrelevanten Bereiche für Frauen abdeckt. Es ist quasi ein völkerrechtliches Grundgesetz für Frauen. Durch seinen sehr umfassenden Begriff der Diskriminierung werden direkte, indirekte und auch strukturelle Diskriminierungen erfasst. Hiernach ist zur Feststellung einer Diskriminierung nicht zwingend erforderlich, einen Vergleich zwischen Frauen und Männern vorzunehmen, sondern es werden alle Praktiken erfasst, die Frauen daran hindern, ihre Menschenrechte auszuüben ${ }^{6}$. Dies macht CEDAW zu einem so wertvollen Instrument gegen die zahlreichen nach wie vor bestehenden Diskriminierungen von Frauen in Deutschland. Das Abkommen hat als völkerrechtli- cher Vertrag in Deutschland gemäß Art. 59 Abs. 2, S. 1 GG über das Ratifikationsgesetz den Rang eines einfachen Gesetzes. Das bedeutet, bei Normenkollisionen mit anderen bundesdeutschen Gesetzen gelten die allgemeinen Grundsätze, insbesondere diejenigen über den Vorrang des spezielleren gegenüber dem generelleren und des jüngeren gegenüber dem älteren $\mathrm{Gesetz}^{7}$. Aus dem Gleichstellungsgebot des Art. 3 CEDAW folgt daher zum Beispiel eine Verpflichtung Deutschlands zum Gender Mainstreaming oder auch zur Durchführung von Bildungs- und Aufklärungskampagnen ${ }^{8}$. In diesem Zusammenhang ist die Auflösung der innerministeriellen „Arbeitsgruppe zur Begleitung des Prozesses von Gender Mainstreaming in allen Bundesministerien “, ohne dass ein gleichwertiger Ersatz geschaffen wurde, nochmals besonders kritisch zu bewerten?.

Gleichstellung von Frauen und Männern als Querschnittsaufgabe in allen Politikbereichen

Die abschließenden Betrachtungen ${ }^{10}$ des CEDAWVertragsausschusses, in denen die Ergebnisse der

1 Mit dem dritten der acht Millenniumsziele haben sich die Teilnehmer/innen dazu verpflichtet, die Gleichstellung der Geschlechter und die politische, wirtschaftliche und soziale Beteiligung von Frauen zu fördern und außerdem erklärt, dass die Gleichstellung der Geschlechter Voraussetzung aller Millenniumsziele ist.

2 Veröffentlicht in BGBI 1985 II, S. 648 ff.

3 In der DDR trat CEDAW bereits 1980 in Kraft.

4 BT-Drs. 16/5807 vom 8.6.2007.

5 Am 2.2.2009 in Genf.

6 S. hierzu ausführlich Beate Rudolf, djbZ 2009, S. 4 f.

7 Rudolf, in Rudolf/Mahlmann, Gleichbehandlungsrecht, 2007, S. 60.

8 So auch Rudolf, a.a.O., S. 68.

9 Auch der CEDAW-Ausschuss mahnt die Bundesregierung, ,zu dem integrierten Konzept der Geschlechtergerechtigkeit zurückzukehren, das in den vorherigen abschließenden Bemerkungen vom Ausschuss begrüßt worden war".

10 Statt "concluding comments “ verwendet der Vertragsausschuss nunmehr den Begriff „,concluding observations".

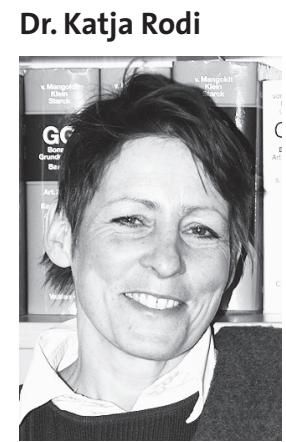

Vorsitzende der Kommission Öffentliches Recht, Europa- und Völkerrecht des djb; Wiss. Mitarbeiterin, Universität Greifswald 
Verhandlungen mit der deutschen Delegation am Anfang dieses Jahres zusammengefasst und gleichzeitig Forderungen des Ausschusses an die deutsche Politik für die folgende Berichtsperiode formuliert wurden, verdeutlichen noch einmal, dass zwar sehr wichtige Einzelmaßnahmen in der deutschen Gleichstellungspolitik zu finden sind ${ }^{11}$, dass aber eine Gesamtkonzeption dieser Politik nicht erkennbar und auch die Wirksamkeit dieser Maßnahmen nur unzureichend untersucht wird. Darüber hinaus wird in den abschließenden Betrachtungen des Ausschusses ein mangelhaftes Zusammenwirken zwischen den einzelnen Bundesministerien und auch zwischen den einzelnen föderalen Ebenen - das heißt zwischen Bundes-, Landes und kommunaler Ebene - festgestellt. Der djb hat als Reaktion auf die Verhandlungen vor dem CEDAW-Vertragsausschuss im März diesen Jahres einen offenen Brief an die Ministerin für Frauen und Familie und gleichzeitig an alle anderen Bundesministerien in Kopie geschickt ${ }^{12}$. In ihren Antworten haben sich die anderen Bundesministerien mit wenigen Ausnahmen ${ }^{13}$ für nicht zuständig erklärt und auf die ausschließliche Kompetenz des Ministeriums für Familie, Senioren, Frauen und Jugend für diese Fragen verwiesen. Gleichstellungs- und Frauenförderpolitik muss aber als Querschnittsaufgabe in allen Fachministerien wahrgenommen werden! Dieses scheint mit wenigen Ausnahmen noch nicht im Bewusstsein der Ministerien angekommen zu sein. Gerade die Ministerien, deren Politik auf der internationalen Ebene besonders kritisch ins Visier genommen wurden - das Wirtschafts- und das Arbeits- und Sozialministerium - definieren Geschlechtergleichstellung oder Frauenförderpolitik zumindest in der Präsentation ihrer Ministerien im Internet nicht als einen ihrer Aufgabenbereiche. Auch in den Bezeichnungen der Abteilungen oder der Referate lässt sich eine Beschäftigung mit diesen Fragestellungen nicht erkennen und zu CEDAW finden sich auf den Internetseiten dieser Ministerien keinerlei Informationen oder auch nur Verlinkungen auf andere Seiten.

\section{Internationale Kritik an der Politik der Bundesregierung}

Vergleicht man die abschließenden Bemerkungen des CEDAW-Vertragsausschusses aus der letzten Berichtsperiode mit den Ergebnissen der diesjährigen Verhandlungen, so wird deutlich, dass sich eine Vielzahl der Kritikpunkte wiederholen. So heißt es, um ein Beispiel herauszugreifen, bereits 2004: „Der Ausschuss fordert den Vertragsstaat auf, seine Anstrengungen zur Förderung der De-facto-Gleichstellung von Frauen auf dem Arbeitsmarkt einschließlich ihres Zugangs zur Vollbeschäftigung zu verstärken ..., und den Grundsatz „gleicher Lohn für gleichwertige Arbeit“ zu fördern. Der Ausschuss empfiehlt dem Vertragsstaat, die Auswirkungen der Vorschriften zur Teilzeitarbeit und Elternzeit weiter zu beobachten und ggf. die Anreize zu verstärken, um möglichen nachteiligen Folgen von Teilzeitarbeit für Frauen entgegenzuwirken, ... “14. Diese Kritik wird in den aktuellen abschließenden Betrachtungen fast unverändert aufgegriffen und weiter verstärkt. Unter der Überschrift „Gleichberechtigte Teilhabe von Frauen am Arbeitsmarkt“ fordert der Ausschuss „den Vertragsstaat ein- dringlich auf, das Allgemeine Gleichbehandlungsgesetz entsprechend zu ändern, damit es dem Übereinkommen voll und ganz entspricht" 15 . Ähnlich scharf sind die nachfolgenden Ausführungen zur Segregation der Arbeitmärkte, zu den Auswirkungen der Arbeitsmarktreformen und zum Rentenrecht. Die Bundesrepublik wird ausdrücklich aufgefordert, „die Auswirkungen des Vierten Gesetzes für moderne Dienstleistungen am Arbeitsmarkt auf die Situation der Frauen zu untersuchen und korrektive Maßnahmen zu ergreifen, darunter die Überprüfung des Konstrukts der „Bedarfsgemeinschaften“16. Vor diesem Hintergrund ist die Antwort der Bundesregierung auf eine entsprechende kleine Anfrage aus dem Bundestag: „Daher besteht aus Sicht der Bundesregierung keine Notwendigkeit, das Konstrukt der Bedarfsgemeinschaft zu überprüfen. “17 nicht mehr nachvollziehbar. Neben diesen kritischen Anmerkungen zur Arbeitsmarkt- und Sozialpolitik der Bundesregierung setzt sich der CEDAW-Ausschuss unter anderem mit der unzureichenden Beteiligung der Länder- und Kommunalebene am Berichtsprozess und der unzureichenden Darstellung dieser Ebenen im Staatenbericht, mit der Vereinbarkeit von Beruf und Privatleben, der politischen Teilhabe von Frauen im öffentlichen Leben, dem Problem von Gewalt gegen Frauen und der multiplen Diskriminierung von Frauen mit Migrationshintergrund auseinander ${ }^{18}$.

\section{Forderung nach einem Aktionsplan Geschlechtergleichstellung}

Diese Zusammenfassung der Ergebnisse aus der letzten CEDAW-Berichtsperiode verdeutlicht, dass die neue Bundesregierung ihre Gleichstellungspolitik umstellen muss, um ihren völkerrechtlichen Verpflichtungen nachzukommen. Eine zusammenfassende Rückschau auf die vorhergehenden CEDAW-Berichtsperioden zeigt darüber hinaus, dass es in den relevanten Bereichen trotz einiger Anstrengungen der Bundesregierungen wenig nennenswerte Fortschritte gegeben hat. So wird das Problem der Lohnlücke zwischen Frauen und Männern seit den siebziger Jahren diskutiert, ohne dass sich hier substanziell etwas verbessert hätte. Daher drängt sich aus Sicht unseres Verbandes die Erarbeitung eines Aktionsplanes Geschlech-

11 Genannt werden können hier vor allem die auch in den letzten abschließenden Bemerkungen des CEDAW-Ausschusses erwähnten Maßnahmen, wie die Verabschiedung des AGG mit seiner Definition der direkten und indirekten Diskriminierung, das einkommensunabhängige Elterngeld mit seinen Partnermonaten, der angestrebte Ausbau der Kinderbetreuungsplätze und der 2. Aktionsplan zur Bekämpfung der Gewalt gegen Frauen.

12 Siehe www.djb.de/Kommissionen/kommission-oeffentlichesrecht-europa-und-voelkerrecht/St_o9-05_Offener-Brief-CEDAW.

13 Inhaltlich antworteten lediglich das Bundesministerium für Bildung und Forschung und das Bundesministerium für Entwicklung und Zusammenarbeit.

14 Anmerkung 25 aus der zosten Sitzung des Ausschusses vom 12. bis 30. Januar 2004.

15 Anmerkung 36 aus der 43sten Sitzung vom 19. Januar bis 6 . Februar 2009.

16 Anmerkung 38 aus der 43sten Sitzung vom 19. Januar bis 6 . Februar 2009.

17 BT-Drs. 16/12838 vom 5.5.2009, S. 4.

18 Siehe hierzu auch die Zusammenfassung der abschließenden Bemerkungen unter www.djb.de/Kommissionen/kommissionoeffentliches-recht-europa-und-voelkerrecht/cedaw/cedawB/. 
tergleichstellung nahezu auf. In mehreren europäischen Ländern gibt es solche Aktionspläne ${ }^{19}$ oder ihre Aufstellung wird wie in Österreich gerade diskutiert. In einem deutschen Aktionsplan Geschlechtergleichstellung sollten auf der Grundlage der Ergebnisse der Ausschussverhandlungen alle Akteurinnen und Akteure (Vertreterinnen und Vertreter aller Bundesministerien, der Länder und Kommunen, aber auch alle anderen öffentlichen und privaten Institutionen und Stellen, deren Aufgabe das Realisieren der Gleichstellung von Frau und Mann ist, insbesondere Nichtregierungsorganisationen, die Arbeitgebenden, Gewerkschaften, Bildungsinstitutionen und Medien) zusammenwirken. Hiermit könnte auch einem weiteren Kritikpunkt aus den abschließenden Betrachtungen des CEDAWAusschusses begegnet werden, in dem eine bessere Zusammenarbeit mit den jeweiligen Nichtregierungsorganisationen bei dem CEDAW-Berichtsprozess angemahnt wurde. Dieser Aktionsplan muss alle Bereiche der UN-Frauenrechtskonven- tion umfassen. Er hat Schwerpunkte und konkrete Ziele für die zukünftige Gleichstellungspolitik in Deutschland (Bundes-, Länder- und kommunale Ebene) und Umsetzungsstrategien mit detaillierten Zuständigkeiten, Umsetzungszeitpunkten und Kostenfolgen zu regeln. Bisherige Maßnahmen müssen bewertet und in den Plan eingegliedert werden. Hierzu gehören auch eine Bewertung der Arbeit der Antidiskriminierungsstelle und die bisher als mangelhaft empfundene Zusammenarbeit dieser Stelle mit den Frauenverbänden.

Die Konvention fordert nicht nur die Abgabe von Regierungsberichten, sondern ist als ein Prozess zu verstehen, in dem alle Betroffenen zusammenwirken sollen (Follow-up-Prozess). Genau dies würde ein Aktionsplan leisten.

19 So z.B. in der Schweiz, in Luxemburg, in Schweden zur Umsetzung der UN-Resolution 1325 oder der Aktionsplan für GM in den Niederlanden.

\section{Systematische Gleichstellungspolitik braucht einen verbindlichen Aktionsplan}

\section{Forderungen des Deutschen Juristinnenbunds an die Bundesregierung}

Der Deutsche Juristinnenbund fordert die neue Bundesregierung auf, im Rahmen eines „Aktionsplans Gleichstellung“ mittels wirksamer gesetzlicher Regelungen für die tatsächliche Durchsetzung der Geschlechtergerechtigkeit zu sorgen und Benachteiligungen wegen des Geschlechts in Beruf, Familie und Gesellschaft abzubauen. Dies gebietet zum einen die Verfassung und zum anderen das Übereinkommen der Vereinten Nationen zur Beseitigung jeder Form von Diskriminierung der Frau (CEDAW), welches nach seiner Ratifikation durch die Bundesregierung vor 24 Jahren geltendes Recht ist. Nach Form und Inhalt bietet sich dieses Übereinkommen geradezu als „roadmap“ zur Gleichberechtigung der Geschlechter an. Die Tatsache, dass mit Blick auf die internationale Geltung des Abkommen einige der Verpflichtungen in der Bundesrepublik bereits eingelöst sind, sollte nicht darüber hinwegtäuschen, dass noch sehr viele Bereiche offen sind. Dazu zählen die Bereiche

1. „Entgeltgleichheit“: hier muss der Gesetzgeber einen Rahmen setzen für geschlechtergerechte Arbeitsbewertung und für geschlechtergerechte Tarifverträge, damit Deutschland auch auf diesem Gebiet im europäischen Vergleich bestehen kann.

2. „Ehegattenbesteuerung“: der „German Sonderweg“ des Ehegattensplittings wirkt als faktisches Zugangshemmnis zur Erwerbstätigkeit während der Ehe und ist zugunsten einer eheunabhängigen Besteuerung nach der individuellen Leistungsfähigkeit, welche familiale Unterhaltspflichten angemessen berücksichtigt, abzuschaffen.
3. „Soziale Sicherheit“: Mini-Jobs und die strukturellen Diskriminierungen in der gesetzlichen Rentenversicherung verhindern die eigenständige Existenzsicherung der Frauen im Alter und führen zwangsläufig in die Altersarmut.

4. „Gesundheit“: nach wie vor gibt es keine gender-sensitive und damit zielgenaue Gesundheitsversorgung. Zielgenauigkeit ist Voraussetzung für effizienten Mitteleinsatz. Der von Gesundheitsökonomen geforderte Wettbewerb der Leistungserbringer um kostengünstige Versorgung setzt eine Ausrichtung auf den tatsächlichen Bedarf voraus, welcher das soziale Geschlecht und das Lebensalter zwingend berücksichtigen muss.

5. Infrastruktur: staatliche Daseinsvorsorge mit direkter Wirkung für die Geschlechtergerechtigkeit darf auch in der Weltwirtschaftskrise nicht auf der Strecke bleiben. Bildungseinrichtungen mit zeitgemäßen und anspruchsvollen Angeboten für Menschen aller Altersstufen sind kein disponibler Luxus, sondern als Grundlage einer wettbewerbsfähigen Gesellschaft bedarfsgerecht auszubauen.

Die Vielzahl dieser Forderungen, die seit Jahren von der Politik vernachlässigt wurden, erfordert einen strukturierten Umsetzungsprozess, der auch Wechselwirkungen einzelner Maßnahmen berücksichtigt und Wertungswidersprüche eliminiert. Dazu sollte die künftige Bundesregierung einen „Aktionsplan Gleichstellung" ins Leben rufen. Der Deutsche Juristinnenbund steht gern beratend zur Verfügung. 